\title{
Photo-production of scalar particles in the field of a circularly polarized laser beam
}

\author{
S. Villalba-Chávez, C. Müller \\ Institut für Theoretische Physik I, Heinrich-Heine-Universität Düsseldorf, Universitätsstr. 1, 40225 Düsseldorf, Germany
}

\begin{abstract}
The photo-production of a pair of scalar particles in the presence of an intense, circularly polarized laser beam is investigated. Using the optical theorem within the framework of scalar quantum electrodynamics, explicit expressions are given for the pair production probability in terms of the imaginary part of the vacuum polarization tensor. Its leading asymptotic behavior is determined for various limits of interest. The influence of the absence of internal spin degrees of freedom is analyzed via a comparison with the corresponding probabilities for production of spin-1/2 particles; the lack of spin is shown to suppress the pair creation rate, as compared to the predictions from Dirac theory. Potential applications of our results for the search of minicharged particles are indicated.
\end{abstract}

'Keywords: Vacuum Polarization, Laser Fields, Particle Production, Spin Effects.

PACS: 12.20.Ds, 11.10.Jj, 13.40-f, 32.80.Wr.

\section{Introduction}

Understanding the nonlinear and unstable nature of the quantum vacuum in the presence of a strong electromagnetic field constitutes an important task of theoretical physics. Corresponding studies have revealed a nontrivial vacuum structure, suitable to explore the low-energy frontier of particle physics [1, 2, 3]. Moreover, perspectives of achieving ultrahigh field intensities $\left(I \sim 10^{26} \mathrm{~W} / \mathrm{cm}^{2}\right)$ in short laser pulses of few femtoseconds duration [4, 5] have motivated a growing interest in the phenomenology purely associated with the quantum nature of the electromagnetic interaction (see [6, 7] for recent reviews). This is because the envisaged laser field strengths lie only 12 orders of magnitude below the critical value $E_{\mathrm{c}}=1.3 \times$ $10^{16} \mathrm{~V} / \mathrm{cm}$ where QED vacuum nonlinearities become substantial and spontaneous vacuum decay into electron-positron $\left(e^{-} e^{+}\right)$ pairs via the Schwinger mechanism is expected to occur [8, 9, 10].

In combination with an incident high-energy particle, strong laser fields can induce $e^{-} e^{+}$pair production already at intensities available today. In a pioneering experiment at SLAC [11], a multi-GeV photon decayed into a pair while propagating through a moderately intense laser pulse $\left(I \sim 10^{18} \mathrm{~W} / \mathrm{cm}^{2}\right)$. This process, involving the simultaneous absorption of several laser photons, represents a nonlinear version of the well-known Breit-Wheeler reaction [12, 13, 14]. The high-energy non-laser photon originated from Compton backscattering of SLAC's ultrarelativistic electron beam off the laser pulse. In the near future, corresponding studies can be conducted within all-optical setups using laser-accelerated relativistic electrons as projectiles [15]. Other pair production mechanisms may be probed in

Email addresses: selym@tp1.uni-duesseldorf.de (S. Villalba-Chávez), c.mueller@tp1.uni-duesseldorf.de (C. Müller) ultrarelativistic proton-laser collisions $[16,17,18,19,20]$.

In view of the upcoming high-field laboratories [4, 5], theoreticians are currently investigating further properties and applications of photo-induced $e^{-} e^{+}$pair production in intense laser fields. For example, due to their broad frequency composition, laser pulses of ultrashort duration have been shown to modify the created particle spectra [21] and lead to characteristic enhancements in the pair production probability [22, 23]. Photoinduced pair production also plays a crucial role for the development of QED cascades which may give rise to $e^{-} e^{+}$plasmas of very high density [24, 25]. The nonlinear Breit-Wheeler process moreover offers a promising means to measure ultrashort $\gamma$-ray pulses via $e^{-} e^{+}$streaking [26]. Superimposing the field of a high-energy photon onto a strong electric field may also help catalyzing the Schwinger effect [27, 28, 29].

In the present Letter, we study the photo-induced creation of a pair of spin-0 particles in the presence of a strong monochromatic laser beam. Our motivation is twofold. First, while the probabilities for the creation of fermion pairs are known for a long time [13, 14], it is relevant to establish the corresponding formulas for scalar particles because they can be useful for the ongoing search of minicharged particles which may have either fermionic or bosonic character [2, 3, 30]. Second, our results provide insights into the fundamental question as to how the spin degree of freedom affects the photo-induced pair production process. To this end, a comparison with the known results for fermion pair production will be drawn. Such an information complements previous works where spin-resolved calculations of the nonlinear Breit-Wheeler process via helicity amplitudes [31] and the internal spin polarization vector [32] were performed. We note besides that comparative studies between the behavior of bosonic and fermionic particles in strong laser fields have recently been carried out with respect to Compton, Mott and Kapitza-Dirac scattering [6, 33, 34], nonlinear 
Bethe-Heitler pair creation in proton-laser collisions [35] and the Klein paradox [36, 37].

Our theoretical approach relies on the polarization tensor $\Pi_{\mu \nu}\left(k_{1}, k_{2}\right)$, of scalar Quantum Electrodynamics (in the oneloop approximation) in the presence of a strong laser field [38, 39] whose imaginary part is related to the pair production probability of scalar particles via the optical theorem. While the polarization tensor for Dirac fermions has already been exploited successfully to calculate various $e^{-} e^{+}$pair production processes in strong laser fields [18, 19, 38], to the best of our knowledge the present calculations represent the first application of the corresponding polarization tensor for the scalar case.

\section{General considerations}

To begin with, let us consider the field of a plane electromagnetic wave of the form 1

$$
\mathscr{A}^{\mu}(x)=a_{1}^{\mu} \psi_{1}(\varkappa x)+a_{2}^{\mu} \psi_{2}(\varkappa x),
$$

with $a_{1,2}$ denoting the wave amplitudes and $\psi_{1,2}$ being arbitrary functions. The wave four-vector $\varkappa^{\mu}=\left(\varkappa^{0}, \varkappa\right)$ fulfills the relations $\varkappa^{2}=0$ and $\varkappa a_{1}=\varkappa a_{2}=a_{1} a_{2}=0$. According to [38, 39], the vacuum polarization tensor in this field,

$$
\begin{aligned}
\Pi^{\mu v}\left(k_{1}, k_{2}\right)= & c_{1} \Lambda_{1}^{\mu} \Lambda_{2}^{v}+c_{2} \Lambda_{2}^{\mu} \Lambda_{1}^{v}+c_{3} \Lambda_{1}^{\mu} \Lambda_{1}^{v} \\
& +c_{4} \Lambda_{2}^{\mu} \Lambda_{2}^{v}+c_{5} \Lambda_{3}^{\mu} \Lambda_{4}^{v}
\end{aligned}
$$

can be expanded in terms of a basis set of Lorentz covariant vectors $\Lambda_{i}^{\mu}$ which are constructed from fundamental symmetry principles. They are explicitly given by

$$
\begin{array}{ll}
\Lambda_{1}^{\mu}(k) & =-\frac{\mathscr{F}_{1}^{\mu v} k_{v}}{(k \varkappa)\left(-a_{1}^{2}\right)^{1 / 2}}, \quad \Lambda_{2}^{\mu}(k)=-\frac{\mathscr{F}_{2}^{\mu v} k_{v}}{(k \varkappa)\left(-a_{2}^{2}\right)^{1 / 2}}, \\
\Lambda_{3}^{\mu}(k) & =\frac{\varkappa^{\mu} k_{1}^{2}-k_{1}^{\mu}(k \varkappa)}{(k \varkappa)\left(k_{1}^{2}\right)^{1 / 2}}, \quad \Lambda_{4}^{\mu}(k)=\frac{\varkappa^{\mu} k_{2}^{2}-k_{2}^{\mu}(k \varkappa)}{(k \varkappa)\left(k_{2}^{2}\right)^{1 / 2}} .
\end{array}
$$

Here $\mathscr{F}_{i}^{\mu \nu}=\varkappa^{\mu} a_{i}^{v}-\varkappa^{v} a_{i}^{\mu}(i=1,2)$ are the amplitudes of the external field modes whereas $k_{1}$ and $k_{2}$ denote the incoming and outgoing four-momenta of the probe photons, respectively. We note that the short-hand notation $k$ in Eq. (3) may stand for either $k_{1}$ or $k_{2}$. It is worth mentioning at this point that, for $k=k_{1}$, the vectors $\Lambda_{1}\left(k_{1}\right), \Lambda_{2}\left(k_{1}\right)$ and $\Lambda_{3}\left(k_{1}\right)$ are orthogonal to each other, $\Lambda_{i}^{\mu}\left(k_{1}\right) \Lambda_{j \mu}\left(k_{1}\right)=-\delta_{i j}$, and fulfill the completeness relation $g^{\mu \nu}-\frac{k_{1}^{\mu} k_{1}^{v}}{k_{1}^{2}}=-\sum_{i=1}^{3} \Lambda_{i}^{\mu}\left(k_{1}\right) \Lambda_{i}^{v}\left(k_{1}\right)$ with $g_{\mu \nu}=\operatorname{diag}(+1,-1,-1,-1)$ denoting the metric tensor. A similar statement applies if the set of vectors $\Lambda_{1}\left(k_{2}\right), \Lambda_{2}\left(k_{2}\right)$ and $\Lambda_{4}\left(k_{2}\right)$ are considered. We emphasize that Eq. (2) does not depend on which choice of $k$ is taken since the difference between $k_{1}$ and $k_{2}$ is proportional to $\varkappa$ [see Eq. (5) below].

The form factors $c_{i}$ in Eq. (2) are distribution-valued functions which depend on the field shape via the functions $\psi_{i}$. They have been evaluated thoroughly for the case of spin- $\frac{1}{2}$ particles

${ }^{1}$ From now on "natural" and Gaussian units $c=\hbar=4 \pi \epsilon_{0}=1$ are used. in [38, 39]. Also for the case when the virtual charge carriers in the Feynman loop are spin-0 particles general expressions for the $c_{i}$ were provided in these references; but these formulas were not further evaluated.

Using the general expressions from [38, 39] and assuming that the laser field is an elliptically polarized wave with

$$
\psi_{1}=\cos (\varkappa x) \quad \text { and } \quad \psi_{2}=\sin (\varkappa x)
$$

we find that the form factors in Eq. (2) for the scalar case are given by

$$
\begin{aligned}
c_{i} & =-i \frac{\alpha}{\pi} m^{2} \int_{-1}^{1} d v \int_{0}^{\infty} \frac{d \rho}{\rho} e^{-\frac{2 i \rho}{|\lambda|\left(1-v^{2}\right)}\left(1+A\left(\xi_{1}^{2}+\xi_{2}^{2}\right)-\frac{k_{1}^{2}\left(1-v^{2}\right)}{4 m^{2}}\right)} \\
& \left.\times(2 \pi)^{4}\left[\delta^{4}\left(k_{1}-k_{2}\right) d_{i}^{(0)}+\sum_{\substack{N=-\infty \\
N \neq 0}}^{\infty} \delta^{4}\left(k_{1}-k_{2}-2 N \varkappa\right) d_{i}^{(N)}\right\rceil\right)
\end{aligned}
$$

Here, $\alpha=e^{2}$ is the fine structure constant, $e$ and $m$ denote the particle charge and mass, respectively, and

$$
\lambda=\frac{\varkappa k}{2 m^{2}}, \quad \xi_{i}^{2}=-\frac{e^{2} a_{i}^{2}}{m^{2}} \quad(i=1,2) .
$$

As Eq. (5) shows, the polarization tensor decomposes into elastic $\left(k_{1}=k_{2}\right)$ and inelastic $\left(k_{1} \neq k_{2}\right)$ parts. Those terms which contain the Dirac deltas $\delta^{4}\left(k_{1}-k_{2}+2 N \varkappa\right)$ with $N \neq 0$ are responsible for the inelastic scattering of a photon in the field of the wave. For our purposes, however, only the elastic part is relevant. The corresponding functions $d_{i}^{(0)}, i=1,2,3,4,5$ contained in Eq. (5) are given by

$$
\begin{gathered}
d_{1}^{(0)}=-d_{2}^{(0)}=\xi_{1} \xi_{2} \rho A_{0} J_{0}(z) \operatorname{sign}[\lambda], \\
d_{3}^{(0)}=-\frac{1}{2} \xi_{1}^{2} A_{1}\left(J_{0}(z)-i J_{0}^{\prime}(z)\right)+\frac{\xi_{1}^{2}}{2} \sin ^{2}(\rho) J_{0}(z) \\
+\frac{i|\lambda|\left(1-v^{2}\right)}{8 \rho}\left(J_{0}(z)-e^{i y}\right), \\
d_{4}^{(0)}=d_{3}^{(0)}\left(\xi_{1}^{2} \leftrightarrow \xi_{2}^{2}\right), d_{5}^{(0)}=-\frac{k_{1}^{2}}{8 m^{2}} v^{2}\left(J_{0}(z)-e^{i y}\right)
\end{gathered}
$$

where $J_{0}(z)$ is the Bessel function of zero order and $J_{0}^{\prime}(z)$ its derivative. The remaining parameters are

$$
\begin{gathered}
A=\frac{1}{2}\left(1-\frac{\sin ^{2}(\rho)}{\rho^{2}}\right), \quad A_{0}=\frac{1}{2}\left(\frac{\sin ^{2}(\rho)}{\rho^{2}}-\frac{\sin (2 \rho)}{2 \rho}\right), \\
z=\frac{2 \rho A_{0}}{1-v^{2}} \frac{\xi_{1}^{2}-\xi_{2}^{2}}{|\lambda|}, \quad y=\frac{2 \rho A}{1-v^{2}} \frac{\xi_{1}^{2}+\xi_{2}^{2}}{|\lambda|},
\end{gathered}
$$

and $A_{1}=A+2 A_{0}$.

A substantial simplification is achieved when the external field is taken as a circularly polarized wave $\left(\xi_{1}=\xi_{2}=\xi\right)$. In this case, we find it convenient to express the elastic contribution as

$$
\Pi_{\mu \nu}^{\text {(elast })}\left(k_{1}, k_{2}\right)=i(2 \pi)^{4} \delta^{4}\left(k_{1}-k_{2}\right) \Pi_{\mu \nu}\left(k_{2}\right)
$$

with

$$
\begin{aligned}
\Pi^{\mu \nu}\left(k_{2}\right)= & \left(\Lambda_{1}^{\mu} \Lambda_{2}^{v}-\Lambda_{2}^{\mu} \Lambda_{1}^{v}\right) \pi_{1}^{(0)}+\left(\Lambda_{1}^{\mu} \Lambda_{1}^{v}+\Lambda_{2}^{\mu} \Lambda_{2}^{v}\right) \pi_{3}^{(0)} \\
& +\Lambda_{3}^{\mu} \Lambda_{3}^{v} \pi_{5}^{(0)} .
\end{aligned}
$$




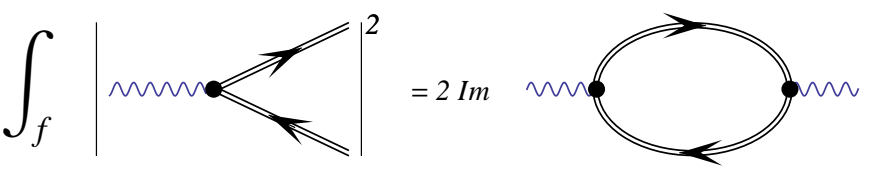

Figure 1: Diagrammatic representation of the optical theorem applied to the photo-production of a pair of spinless particles in the field of a wave. In the left-hand side we have represented the squared modulus of the the $S$-matrix element integrated over the final phase volume $\int_{f} \equiv \int \frac{d^{3} p_{+}}{(2 \pi)^{3}} \frac{d^{3} p_{-}}{(2 \pi)^{3}}$. Here the double lines refer to the exact Klein-Gordon states interacting with the external background. The right-hand side, however, contains the imaginary part of the vacuum polarization tensor where the double lines represent the exact propagators in the field of the wave. In both sides the wavy lines denote the legs corresponding to a photon field.

Here the involved coefficients are given by

$$
\pi_{i}^{(0)}=-\frac{\alpha}{2 \pi} m^{2} \int_{-1}^{1} d v \int_{0}^{\infty} \frac{d \rho}{\rho} e^{-\frac{2 i \rho}{|\lambda|\left(1-v^{2}\right)}\left(1+2 A \xi^{2}-\frac{k_{2}^{2}\left(1-v^{2}\right)}{4 m^{2}}\right)} \Omega_{i}^{(0)}
$$

where

$$
\begin{aligned}
& \Omega_{1}^{(0)}=2 \xi^{2} A_{0} \rho \operatorname{sign}[\lambda], \Omega_{5}^{(0)}=-\frac{k_{2}^{2}}{4 m^{2}} v^{2}\left(1-e^{i y}\right), \\
& \Omega_{3}^{(0)}=\xi^{2} \sin ^{2}(\rho)+\frac{1}{2}\left[1-\frac{k_{1} k_{2}}{4 m^{2}}\left(1-v^{2}\right)\right]\left(1-e^{i y}\right) .
\end{aligned}
$$

Now, the unitarity condition of the dispersion $S$-matrix provides the optical theorem. According to the latter, the total creation rate of a pair of spin-0 particles from a real photon $\left(k^{2}=0\right)$ with polarization vector $\epsilon_{\ell}^{\mu}(\ell=1,2)$ turns out to be $\mathfrak{R}_{\ell}^{(0)}=\epsilon_{\ell}^{\mu} \epsilon_{\ell}^{* v} \operatorname{Im} \Pi_{\mu v} / \omega$ where $\omega$ is the photon frequency (see Fig. 1). If the photon is unpolarized, the averaged rate $\mathfrak{R}^{(0)}=\left(\mathfrak{R}_{1}^{(0)}+\mathfrak{R}_{2}^{(0)}\right) / 2$ reads

$$
\mathfrak{R}^{(0)}=\sum_{\ell} \frac{\epsilon_{\ell}^{\mu} \epsilon_{\ell}^{* v}}{2 \omega} \operatorname{Im} \Pi_{\mu v}(k)=-\frac{g^{\mu v}}{2 \omega} \operatorname{Im} \Pi_{\mu v}(k),
$$

where the completeness relation $g^{\mu \nu}=-\sum_{\ell} \epsilon_{\ell}^{\mu} \epsilon_{\ell}^{* v}$ was used in the second step. Because of this fact, the expression above reduces to

$$
\mathfrak{R}^{(0)}=\frac{\operatorname{Im} \pi_{3}^{(0)}}{\omega} .
$$

So, among the set of form factors previously described in Eqs. (13)- 15], only one contributes to $\mathfrak{R}^{(0)}$. In correspondence, the probability of producing a pair of scalar particles by a photon traveling through a laser field of circular polarization can be represented by the following double-parametric integral

$$
\begin{aligned}
\mathfrak{R}^{(0)}(\xi, \lambda)=- & \frac{\alpha m^{2}}{2 \pi \omega} \operatorname{Im} \int_{0}^{1} d v \int_{0}^{\infty} \frac{d \rho}{\rho} e^{-\frac{2 i \eta}{1-\nu^{2}}}\left\{1-e^{i y}\right. \\
& \left.+2 \xi^{2} \sin ^{2}(\rho)\right\},
\end{aligned}
$$

where the abbreviation $\eta=(\rho / \lambda)\left[1+\xi^{2}\left(1-\sin ^{2}(\rho) / \rho^{2}\right)\right]$ has been used. In the case under consideration the parameter $y$ of Eq. (10) becomes $y=\frac{2 \xi^{2}}{1-v^{2}} \frac{\rho}{\lambda}\left(1-\sin ^{2}(\rho) / \rho^{2}\right)$. Note that for a real non-laser photon (with $k^{2}=0$, i.e. $\omega=|\boldsymbol{k}|$ ) the parameter $\lambda$ in Eq. (6) is always nonnegative $\lambda \geqslant 0$.
The structure of Eq. (18) shares certain similarities with the corresponding production rate $\mathfrak{R}^{\left(\frac{1}{2}\right)}$ of spin- $\frac{1}{2}$ particles which is given by [39]:

$$
\begin{gathered}
\Re^{\left(\frac{1}{2}\right)}(\xi, \lambda)=\frac{\alpha m^{2}}{\pi \omega} \operatorname{Im} \int_{0}^{1} d v \int_{0}^{\infty} \frac{d \rho}{\rho} e^{-\frac{2 i \eta}{1-v^{2}}}\left\{1-e^{i y}\right. \\
\left.-2 \xi^{2} \frac{1+v^{2}}{1-v^{2}} \sin ^{2}(\rho)\right\} .
\end{gathered}
$$

Eqs. (18) and 19) differ, however, by (i) an overall factor of -2 which coincides with the spin- $\frac{1}{2}$ multiplicity, and (ii) the precise form of the integral over $v$. As we will see, this gives rise to differences between $\mathfrak{R}^{(0)}$ and $\mathfrak{R}^{\left(\frac{1}{2}\right)}$ regarding both the absolute size and the functional dependence. We should mention at this point that an exact evaluation of $\mathfrak{R}^{(0)}$ is quite difficult to perform. However, the dependence on the parameters of the theory, i.e. $\lambda$ and $\xi$, allows to obtain closed-form analytical rate expressions in various asymptotic regimes of interest. They will be derived in the forthcoming section.

\section{Asymptotic regimes and comparison with spin- $\frac{1}{2}$ case}

Before undertaking the main calculations of this section, we briefly analyze the kinematics associated with the decay process $\gamma(k)+n \gamma_{L}(\varkappa) \rightarrow \ell^{-}+\ell^{+}$, where $n$ denotes the number of participating laser photons $\gamma_{L}$. In the center-of-mass frame, the corresponding energy-momentum balance implies the condition $n k \varkappa=2 \varepsilon^{2}$, with $\varepsilon$ being the (laser-dressed) energy of a final particle state. Accordingly, whenever the number of absorbed laser photons exceeds the threshold value

$$
n_{*}=\frac{2 m_{*}^{2}}{k \varkappa}
$$

the decay can occur. Here, $m_{*} \equiv m\left(1+\xi^{2}\right)^{1 / 2}$ denotes the effective mass of the particle. It comes out as a consequence of considering the dressed four-momentum $q_{\mu}=p_{\mu}+\frac{m^{2} \xi^{2}}{(\varkappa p)} \varkappa_{\mu}$ (with $p_{\mu}$ the free four-momentum of the particle, i.e. $p^{2}=m^{2}$ ) as the kinematical variable involved in the energy-momentum conservation [13, 14]. We point out that in the center-of-mass frame, the relative speed between the created particles is given by $\left|\boldsymbol{v}_{\text {rel }}\right|=\left|\boldsymbol{v}_{-}-\boldsymbol{v}_{+}\right|=2 v$ with

$$
v=\frac{|\boldsymbol{q}|}{\varepsilon}=\left(1-\frac{n_{*}}{n}\right)^{1 / 2} \text {. }
$$

\subsection{Two-photon reaction at $\xi<1$}

We wish to specialize Eq. 18 to the case where $\xi<1$. In this context, we note that the probability of creating a pair is suppressed if the condition $\lambda \ll 1$ holds, so that dispersive processes become of main interest here? 2 . On the contrary, if $\lambda \geqslant 1+\xi^{2}$ (i.e. $n_{*} \leqslant 1$ ), the pair production could take place with the absorption of just one photon from the laser wave (twophoton reaction). In this case, which we shall consider now, the

\footnotetext{
${ }^{2}$ In the aforementioned limit, the theoretical description of such phenomena can be performed by means of an effective Lagrangian approach. For details we refer the reader to [7, 41, 42, 43].
} 
oscillatory contribution present in the exponent of 18 becomes very small in comparison with the remaining terms, allowing to expand $\exp \left[\frac{i \xi^{2}}{\lambda} \frac{\sin ^{2}(\rho)}{\rho}\right]$ without obstruction. Afterwards, we perform a change of variable $\rho \rightarrow \rho\left(1+\xi^{2}\right)$ in the integral which corresponds to the second term inside the curly brackets in Eq. (18) to arrive at

$$
\begin{aligned}
\mathfrak{R}^{(0)} \simeq- & \frac{\alpha m^{2} \xi^{2}}{2 \pi \omega} \int_{0}^{1} d v \int_{-\infty}^{\infty} \frac{d \rho}{\rho} e^{\frac{2 i \rho\left(1+\xi^{2}\right)}{\lambda\left(1-v^{2}\right)}} \sin ^{2}(\rho) \\
& \times\left\{i+\frac{1}{\lambda \rho\left(1-v^{2}\right)}\right\} .
\end{aligned}
$$

In the derivation of this expression a term of the order $\sim \xi^{2} / \lambda$ has been accounted for, whereas terms of higher order in $\xi^{2} / \lambda$ were dropped. Hence, the results obtained in this section apply whenever the following conditions are simultaneously fulfilled:

$$
\lambda \geqslant 1+\xi^{2} \quad \text { and } \quad \lambda \gg \xi^{2} .
$$

Besides, to obtain $\mathrm{Eq}$. (22) the $\operatorname{Im}(. .$.$) present in Eq. 18$ must be carried out, after which the resulting integrand turns out to be an even function in the $\rho$-variable. This symmetry allows to perform the change in the integration limits according to $\int_{0}^{\infty} d \rho \ldots \rightarrow \frac{1}{2} \int_{-\infty}^{\infty} d \rho \ldots$ as well as to express the integrand as it stands in (22).

In order to provide an explicit expression of $\mathfrak{R}^{(0)}$ we integrate by parts the terms containing the factor proportional to $1 / \rho^{2}$. Afterwards, the residue theorem is applied. To this end, the contour of the $\rho$-integration is chosen slightly below the real $\rho$ axis (cf. also [39]). As a consequence we obtain

$$
\begin{aligned}
\mathfrak{R}^{(0)}= & -\frac{\alpha m^{2} \xi^{2}}{2 \omega} \int_{0}^{1} d v \theta\left(1-\frac{1+\xi^{2}}{\lambda\left(1-v^{2}\right)}\right) \\
& \times\left\{\frac{1}{\lambda\left(1-v^{2}\right)}-\frac{1}{2}\left(1+\frac{2\left(1+\xi^{2}\right)}{\lambda^{2}\left(1-v^{2}\right)^{2}}\right)\right\}
\end{aligned}
$$

where $\theta(x)$ denotes the unit step function. The remaining integral over $v$ can be taken analytically without complications. It leads to

$$
\begin{aligned}
\mathfrak{R}^{(0)} \simeq \frac{\alpha m^{2} \xi^{2}}{4 \omega} & {\left[\left(1+\frac{n_{*}}{1+\xi^{2}}\right) \sqrt{1-n_{*}}-\frac{n_{*}}{1+\xi^{2}}\left(1-\frac{1}{2} n_{*}\right)\right.} \\
\times & \left.\ln \left(\frac{1+\sqrt{1-n_{*}}}{1-\sqrt{1-n_{*}}}\right)\right] \theta\left(1-n_{*}\right)
\end{aligned}
$$

with $n_{*}$ given in (20). The respective expression for $\mathfrak{R}^{\left(\frac{1}{2}\right)}$ can be read off from Eq. (24) by multiplying the latter by -2 and replacing the coefficient in front of the logarithmic function by $1+n_{*} /\left(1+\xi^{2}\right)-n_{*}^{2} / 2\left(1+\xi^{2}\right)$ (see Appendix D in [39]).

Let us consider some limiting cases. At $n_{*} \simeq 1$, i.e. in the nonrelativistic limit $(v \ll 1)$, Eq. (24) behaves as

$$
\mathfrak{R}^{(0)} \approx \frac{\alpha m^{2} \xi^{2}}{4 \omega}\left(1-n_{*}\right)^{1 / 2},
$$

whereas $\mathfrak{R}^{\left(\frac{1}{2}\right)}=2 \mathfrak{R}^{(0)}$. So, in this limit, the ratio between the production probabilities coincides with the spin multiplicity of

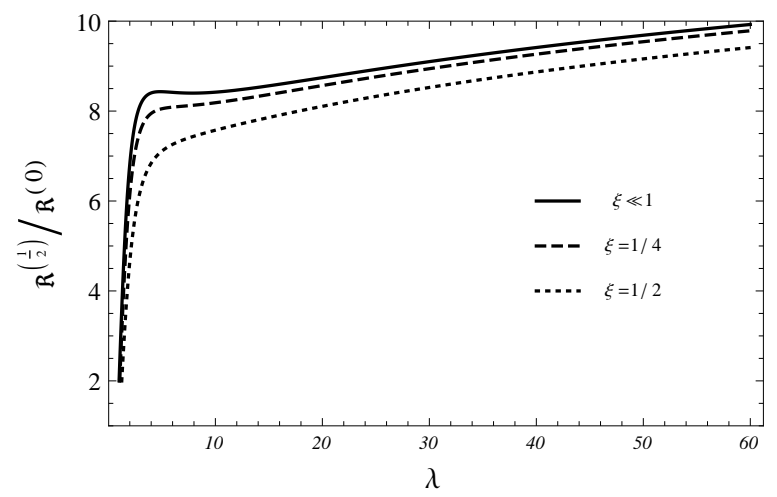

Figure 2: Relative dependence of the fermionic and scalar pair production rates on the parameter $\lambda$ for different values of $\xi<1$, as indicated. The case $\xi \ll 1$ corresponds to the Born approximation.

a Dirac particle. Incidentally, this relation is also manifest to leading order between the rates of the respective Schwinger mechanisms [44]. In contrast, for $n_{*} \approx 0$, the created particles are ultrarelativistic $(v \sim 1)$ and the probability becomes independent of $\lambda$ :

$$
\mathfrak{R}^{(0)} \approx \frac{\alpha m^{2} \xi^{2}}{4 \omega} .
$$

The situation is quite different when the photo-production of Dirac particles is considered. In fact, under the same circumstances, the creation rate of a pair of spin- $\frac{1}{2}$ particles has a logarithmic dependence: $\mathfrak{R}\left(\frac{1}{2}\right) \approx \frac{\alpha m^{2} \xi^{2}}{2 \omega}\left[2 \ln \left(2 \varepsilon / m_{*}\right)-1\right]$; see also [40]. As a consequence, the ratio between the fermionic and scalar rates grows logarithmically, i.e. $\mathfrak{R}^{\left(\frac{1}{2}\right)} / \mathfrak{R}^{(0)} \sim 4 \ln \left(2 \varepsilon / m_{*}\right)$ as $\varepsilon \sim(k \varkappa)^{1 / 2} \gg m_{*}$. Such a behavior resembles the corresponding result arising in Compton scattering. Indeed, as is well known, when the energy of the incoming photon is very large $(\omega \gg m)$ the total Compton cross section computed for Dirac fermions turns out to be $\sigma^{\left(\frac{1}{2}\right)} \approx \frac{\alpha^{2} \pi}{\omega m}[\ln (2 \omega / m)+1 / 2]$. In contrast, the leading asymptotic behavior of the corresponding total cross section determined for spin-0 particles reads $\sigma^{(0)} \approx$ $\frac{2 \alpha^{2} \pi}{\omega m}$ [40]. Accordingly, $\sigma^{\left(\frac{1}{2}\right)} / \sigma^{(0)} \sim \frac{1}{2} \ln (2 \omega / m)$ as $\omega \gg m$. Obviously, in the case under consideration, $\mathfrak{R}^{\left(\frac{1}{2}\right)} / \mathfrak{R}^{(0)}$ can be substantially larger than the statistical factor of 4 associated with the possible spin configurations in the final state.

The results obtained in this section are summarized in Fig. 2 which displays the ratio $\mathfrak{R}^{\left(\frac{1}{2}\right)} / \mathfrak{R}^{(0)}$ as a function of $\lambda$. Several curves are shown corresponding to different values of $\xi$. The picture also includes the limiting case of $\xi \ll 1$ (solid line), which is compatible with the Born approximation. The explicit expressions associated with this limit can be read off from Eqs. (24)-25) by replacing $n_{*} \rightarrow n_{\mathrm{B}}=1 / \lambda$ and setting $\xi=0$ within the squared brackets. The latter procedure leaves us with a quadratic dependence on the parameter $\xi$. Moreover we note that, at larger values of $\xi \approx 1$ and $\lambda \sim 1+\xi^{2}$, next-to-leading order terms become increasingly important (see also [18]). These terms which may give some minor contribution to the rates for $\xi=1 / 2$ have not been included in Fig. 2. 


\subsection{Leading behavior of $\mathfrak{R}^{(0)}$ at asymptotically large $\xi \gg 1$}

In order to undertake the calculations in the high-field domain with $\xi \gg 1$ it is convenient to express the integral of the first two terms in Eq. (18) in a more suitable form:

$$
\begin{aligned}
& \operatorname{Im} \int_{0}^{1} d v \int_{0}^{\infty} \frac{d \rho}{\rho} e^{-\frac{2 i \eta}{\left(1-v^{2}\right)}}\left(1-e^{y}\right) \\
& =-\frac{16 \xi^{2}}{\lambda} \operatorname{Re} \int_{0}^{1} d v \frac{v^{2}}{\left(1-v^{2}\right)^{2}} \int_{0}^{\infty} d \rho A_{0} e^{-\frac{2 i \eta}{\left(1-v^{2}\right)}} \\
& \left.=-\frac{16 \xi^{2}}{\lambda} \int_{0}^{1} \frac{d v v^{2}}{\left(1-v^{2}\right)^{2}} \int_{0}^{\infty} d \rho A_{0} \cos \left(\frac{2 \eta}{\left(1-v^{2}\right)}\right) 27\right)
\end{aligned}
$$

with $A_{0}$ given in Eq. (10) and $\eta$ defined below Eq. (18). Inserting the expression above into Eq. (18) we obtain

$$
\begin{aligned}
\mathfrak{R}^{(0)}=- & \frac{i \alpha m^{2}}{4 \pi \omega} \int_{1}^{\infty} \frac{d u}{2 u[u(u-1)]^{1 / 2}} \int_{-\infty}^{\infty} \frac{d \rho}{\rho} e^{2 i u \eta} \\
& \times\left\{2 \xi^{2} \sin ^{2}(\rho)+\frac{16 i \xi^{2}}{\lambda} u(u-1) \rho A_{0}\right\},
\end{aligned}
$$

where the lower boundary of the $\rho$-integral was extended to $-\infty$ taking into account the symmetry of the integrand in this variable. Besides, the change of variable $u=\left(1-v^{2}\right)^{-1}$ has been carried out. We point out that the integral over this variable does not diverge, although the integrand is a singular function at $u=1$. Since the latter, moreover, decreases very fast like $\sim 1 / u^{2}$ when $u \rightarrow \infty$, it is expected that the main contribution results from the region around $u \sim 1$. The situation is somewhat different with respect to the variable $\rho$. While in this case the integrand falls off for $\rho \rightarrow \pm \infty$ as well, it is a regular function in $\rho$ with vanishing limit at $\rho \rightarrow 0$. In order to elucidate the mainly contributing region of this integral, we split the integration domain into three regions: from $-\infty$ to $-\rho_{0}$ (lower region), from $-\rho_{0}$ to $\rho_{0}$ (inner region) and from $\rho_{0}$ to $\infty$ (upper region). This is,

$$
\int_{-\infty}^{\infty} \frac{d \rho}{\rho} \ldots=\int_{-\infty}^{-\rho_{0}} \frac{d \rho}{\rho} \ldots+\int_{-\rho_{0}}^{\rho_{0}} \frac{d \rho}{\rho} \ldots+\int_{\rho_{0}}^{\infty} \frac{d \rho}{\rho} \ldots
$$

where the positive dimensionless parameter $\rho_{0}$ is chosen to fulfill the conditions

$$
\xi^{-1} \ll \rho_{0} \ll 1 \text { and }\left(\lambda / \xi^{2}\right)^{1 / 3} \ll \rho_{0} \ll 1 .
$$

Within the inner narrow integration region, where $|\rho| \leqslant \rho_{0} \ll 1$, one may Taylor expand $\eta$ in the exponential and, separately, the remaining part of the integrand. Afterwards, we perform a change of variable according to $s=\rho \xi$. The latter is also carried out in the integrals over the lower and upper regions but, after extending the resulting integration limit $\rho_{0} \xi \rightarrow \infty$, no relevant contribution comes from them. Thus, the total integral over $\rho$ can be well approximated by

$$
\begin{array}{r}
\int_{-\infty}^{\infty} \frac{d \rho}{\rho} \ldots \approx \int_{-\infty}^{\infty} d s\left\{2 s \sin \left[\frac{2 u}{\lambda \xi}\left(s+\frac{s^{3}}{3}\right)\right]+\frac{8 u(u-1)}{3 \lambda \xi}\right. \\
\left.\times s^{2} \cos \left[\frac{2 u}{\lambda \xi}\left(s+\frac{s^{3}}{3}\right)\right]\right\} .
\end{array}
$$

We remark that Eq. 30 is accurate up to terms that decrease exponentially, like $\sim\left(\rho_{0} \xi\right)^{-1} \exp \left[-\frac{2 u}{3 \lambda \xi}\left(\rho_{0} \xi\right)^{3}\right]$ or even faster.

The integration over $s$ can, then, be done with the help of the Macdonald function $K_{v}(x)$ using the relations 3

$$
\begin{aligned}
& \int_{-\infty}^{\infty} d y y \sin \left(b y+a y^{3}\right)=\frac{2}{3 \sqrt{3}} \frac{b}{a} K_{2 / 3}\left(\frac{2}{3 \sqrt{3}} \frac{b^{3 / 2}}{a^{1 / 2}}\right), \\
& \int_{-\infty}^{\infty} d y y^{2} \cos \left(b y+a y^{3}\right)=-\frac{2}{9} \frac{b^{3 / 2}}{a^{3 / 2}} K_{1 / 3}\left(\frac{2}{3 \sqrt{3}} \frac{b^{3 / 2}}{a^{1 / 2}}\right) .
\end{aligned}
$$

We insert the resulting expression into Eq. (28) and use the identity $-x K_{1 / 3}(x)=x \frac{d}{d x} K_{2 / 3}(x)+\frac{2}{3} K_{2 / 3}(x)$ (see Eqs. (8.486.12) and (8.486.16) in [45]) to obtain

$$
\mathfrak{R}^{(0)} \simeq \frac{\alpha m^{2}}{12 \sqrt{3} \pi \omega} \int_{1}^{\infty} \frac{d u}{u \sqrt{u(u-1)}} K_{2 / 3}\left(\frac{4 u}{3 \zeta}\right)(4 u-1)
$$

where, in addition, an integration by parts has been carried out. Besides, the abbreviation $\zeta \equiv \lambda \xi=\frac{\omega}{2 m} \frac{E}{E_{c}}(1-\hat{\boldsymbol{k}} \cdot \hat{\boldsymbol{\varkappa}})$ has been introduced, with the critical field strength $E_{c}=m^{2} / e$. The parameter $\zeta$ encodes the two paths to vacuum polarization effects: either by increasing the external field amplitude or by increasing the photon energy. It also depends on the propagation directions of the photon $\hat{\boldsymbol{k}}=\boldsymbol{k} /|\boldsymbol{k}|$ and the strong laser field $\hat{\boldsymbol{\varkappa}}=\varkappa /|\varkappa|$. Observe that $\zeta$ is maximized when the fields counterpropagate, i.e., when $\hat{\boldsymbol{k}} \cdot \hat{\boldsymbol{\varkappa}}=-1$.

We recall that Eq. 33 was derived under the assumptions given in 29]. Thus, the rate expression above applies when the number of absorbed laser photons is very large, $n \geqslant n_{*} \approx$ $\xi^{2} / \lambda \gg 1$. In this case, many photon orders $n$ contribute to the production rate [13, 14]. Note that Eq. (33) is independent of the frequency of the strong background field. The problem thus becomes quasi-static with respect to the external wave which, consequently, may be approximated by a pure constant crossed field. We have checked that, starting from the general expression of the vacuum polarization tensor $\Pi_{\mu \nu}^{(\text {scal })}$ in such an external field configuration [46], we can also arrive at Eq. (33).

The corresponding production rate of spin- $\frac{1}{2}$ particles emerges from (33) via the replacement: $(4 u-1) \rightarrow 2(8 u+1)$. The resulting integrand turns out to be greater than the respective one of Eq. (33) within the whole integration domain of the $u$-variable. This fact guarantees that $\mathfrak{R}^{\left(\frac{1}{2}\right)} / \mathfrak{R}^{(0)}>1$. Hence the internal spin degrees of freedom promote the creation rate of spin- $\frac{1}{2}$ particles as compared with scalar ones.

So far, no restriction has been imposed on the parameter $\zeta$. Let us consider the situation in which $\zeta \gg 1$. To be consistent with our previous condition $(\xi \gg 1)$ the parameter $\lambda$ must be restricted to $\lambda \gg 1 / \xi$. We may exploit the small-argument behavior of the Macdonald functions, $K_{v}(x) \sim \frac{\Gamma[v]}{2}\left(\frac{2}{x}\right)^{v}$ [45], and

\footnotetext{
${ }^{3}$ Equations (3.695.1-2) in Ref. [45] allow to find the identity

$$
\int_{-\infty}^{\infty} d y \cos \left(b y+a y^{3}\right)=\frac{2}{3} \sqrt{\frac{b}{a}} K_{1 / 3}\left(\frac{2}{3 \sqrt{3}} \frac{b^{3 / 2}}{a^{1 / 2}}\right) .
$$
}

After differentiating with respect to $b$ and using Eqs. (8.486.10-11) of the aforementioned reference we establish the relations 31-32. 
obtain

$$
\mathfrak{R}^{(0)} \simeq \frac{\alpha m^{2} \zeta^{2 / 3}}{6 \sqrt{3 \pi} \omega}\left(\frac{3}{2}\right)^{2 / 3} \frac{\Gamma^{2}\left(\frac{2}{3}\right)}{\Gamma\left(\frac{13}{6}\right)},
$$

with $\Gamma(x)$ denoting the Gamma function. The corresponding result for the fermionic case is given in [39]. Here the role of the internal spin degrees of freedom are manifest as $\mathfrak{R}^{\left(\frac{1}{2}\right)}=5 \mathfrak{R}^{(0)}$.

On the contrary, if $\zeta \ll 1$ (corresponding to $\lambda \ll 1$ at $\xi \gg 1$ ), the large asymptotic behavior of the Macdonald functions applies, i.e. $K_{v}(x) \sim \sqrt{\frac{\pi}{2 x}} e^{-x}$ [45]. With this expansion in mind, the integration over $u$ can be performed. The latter becomes particularly simple because the region $u \sim 1$ provides the essential contribution. This leads to

$$
\mathfrak{R}^{(0)} \simeq \frac{\alpha m^{2} \zeta}{16 \omega} \sqrt{\frac{3}{2}} e^{-\frac{4}{3 \zeta}} .
$$

A comparison with the corresponding fermionic rate [40] leads to write $\mathfrak{R}^{\left(\frac{1}{2}\right)}=6 \mathfrak{R}^{(0)}$. It is interesting to note that a similar result was found for the strong-field Bethe-Heitler process $\gamma_{\text {Coul }}+n \gamma_{L} \rightarrow \ell^{-}+\ell^{+}$. In the parameter domain where $\xi \gg 1$ and $E \sim E_{c}$, the rate associated with the creation of Dirac fermions in the Coulomb field of a nucleus exceeds by a factor of $\simeq 7$ the corresponding rate for spin-0 particles [35].

\section{Conclusions and outlook}

Photo-initiated production of a pair of spinless particles in the field of a circularly polarized laser wave was investigated. Compact expressions for the pair production rate were obtained in various asymptotic parameter regimes. Our analysis was carried out by considering the imaginary part of the vacuum polarization tensor as dictated by the optical theorem. Comparisons between the creation rates for spin- $\frac{1}{2}$ versus spin-0 particles revealed how spin effects are manifest at different energy scales. It was shown that the spin degrees of freedom affect the absolute magnitude and, in the limit of high photon energies, also the functional form of the production rates. The rate predictions based on the Dirac theory were generally found to be significantly larger than the corresponding results for Klein-Gordon particles.

We note that the absence of internal spin degrees of freedom in the scalar theory renders the evaluation of the vacuum polarization tensor somewhat easier than in the fermionic case. It is worth mentioning that, since the total production rates for spin-0 and spin- $\frac{1}{2}$ particles in the high-field limit $(\xi \gg 1)$ differ by an overall factor only, this technical simplification could be exploited to extract new insights into the production process which are valid not only for scalar particles but also for $e^{-} e^{+}$ pairs. Indeed, the very similar behavior of the production rates in this regime indicates that the process is mainly determined by the features of the weak photon and strong laser fields, rather than the particular property of the matter field.

Finally we point out that, whenever the energy scale remains within the phenomenological limits of QED and its fundamental principles are preserved, the photo-production of a hypothetical pair of spinless particles characterized by a tiny fraction $\epsilon$ of the electron charge $e$ would not differ qualitatively from the respective creation of a spinless electron-positron pair. In correspondence, the rate associated with the latter phenomenon can be obtained from the expression derived in this Letter by replacing the electron parameters $(e, m)$ by the respective quantities $\left(\epsilon e, m_{\epsilon}\right)$ associated with a minicharged particle. Therefore, our results can be useful in the ongoing search for minicharged particles [1, 2, 3, 30] and help to improve our understanding of how the spin degrees of freedom affect the relevant experimental observables.

\section{Acknowledgments}

S. Villalba-Chávez gratefully acknowledges the support by the Alexander von Humboldt Foundation.

\section{References}

[1] H. Gies. Eur. Phys. J. D 55, 311 (2009); [arXiv:0812.0668 [hep-ph]].

[2] J. Jaeckel and A. Ringwald. Ann. Rev. Nucl. Part. Sci. 60, 405 (2010); arXiv:1002.0329 [hep-ph]].

[3] B. Döbrich, H. Gies, N. Neitz and F. Karbstein. Phys. Rev. Lett. 109, 131802 (2012); [arXiv:1203.2533 [hep-ph]].

[4] See: http://www.extreme-light-infrastructure.eu

[5] See: http://www.hiperlaser.org/index.asp

[6] F. Ehlotzky, K. Krajewska and J. Z. Kamiński. Rep. Prog. Phys. 72, 046401 (2009).

[7] A. Di Piazza, C. Müller, K. Z. Hatsagortsyan and C. H. Keitel Rev. Mod. Phys. 84, 1177 (2012); arXiv:1111.3886 [hep-ph].

[8] F. Sauter. Z. Phys. 69, 742 (1931).

[9] W. Heisenberg and H. Euler. Z. Phys. 98, 714 (1936).

[10] J. S. Schwinger. Phys. Rev. 82, 664 (1951).

[11] D. L. Burke et al. Phys. Rev. Lett. 79, 1626 (1997).

[12] G. Breit and J. A. Wheeler. Phys. Rev. 46, 1087 (1934).

[13] H. R. Reiss. Jour. Math. Phys. 3, 59 (1962).

[14] N. V. Narozhnyi, A. I. Nikishov and V. I. Ritus. [Sov. Phys. JETP 20, $622(1965)]$.

[15] H. Hu, C. Müller and C. H. Keitel. Phys. Rev. Lett. 105, 080401 (2010).

[16] C. Müller, A. B. Voitkiv and N. Grün. Phys. Rev. Lett. 91, 223601 (2003).

[17] P. Sieczka et al. Phys. Rev. A. 73, 053409 (2006).

[18] A. I. Milstein et al. Phys. Rev. A 73, 062106 (2006).

[19] A. Di Piazza, E. Lötstedt, A. I. Milstein and C. H. Keitel. Phys. Rev. Lett. 103, 170403 (2009); arXiv:0906.0726 [hep-ph]].

[20] C. Müller. Phys. Lett. B 672, 56 (2009); arXiv:0811.0976 [hep-ph]].

[21] T. Heinzl, A. Ilderton and M. Marklund. Phys. Lett. B 692, 250 (2010); arXiv:1002.4018 [hep-ph]].

[22] A. I. Titov, H. Takabe, B. Kämpfer and A. Hosaka. Phys. Rev. Lett. 108, 240406 (2012).

[23] T. Nousch, D. Seipt, B. Kämpfer and A. I. Titov. Phys. Lett. B 715, 246 (2012).

[24] A. R. Bell and J. G. Kirk. Phys. Rev. Lett. 101, 200403 (2008).

[25] E. N. Nerush et al. Phys. Rev. Lett. 106, 035001 (2011).

[26] A. Ipp, J. Evers, C. H. Keitel and K. Z. Hatsagortsyan. Phys. Lett. B 702, 383 (2011).

[27] R. Schützhold, H. Gies and G. V. Dunne. Phys. Rev. Lett. 101, 130404 (2008); [arXiv:0807.0754 [hep-th]].

[28] G. V. Dunne, H. Gies and R. Schützhold. Phys. Rev. D 80, 111301 (2009); [arXiv:0901.2631 [hep-ph]].

[29] M. Orthaber, F. Hebenstreit and R. Alkofer. Phys. Lett. B 698, 80 (2011); arXiv:1102.2182 [hep-ph]].

[30] M. Ahlers, H. Gies, J. Jaeckel and A. Ringwald. Phys. Rev. D 75, 035011 (2007); |hep-ph/0612098].

[31] Y. S. Tsai, Phys. Rev. D 48, 96 (1993).

[32] D. Y. Ivanov, G. L. Kotkin and V. G. Serbo. Eur. Phys. J. C 40, 27 (2005); [hep-ph/0412032]. 
[33] M. Boca, V. Dinu and V. Florescu. Nucl. Instrum. Meth. Phys. Res. B 279, 12 (2012).

[34] S. Ahrens, H. Bauke, C. H. Keitel and C. Müller. Phys. Rev. Lett. 109, 043601 (2012).

[35] T. O. Müller and C. Müller. Phys. Lett. B. 696, 201 (2011); arXiv:1007.5176 [hep-ph]].

[36] R. E. Wagner, M. R. Ware, Q. Su and R. Grobe. Phys. Rev. A 81 , 024101 (2010).

[37] P. Krekora, Q. Su and R. Grobe. Phys. Rev. Lett. 92, 040406 (2004).

[38] V. N. Bă̌er, A. I. Mil'shteŭn and V. M. Strakhovenko. Zh. Eksp. Teo. Fiz. 69, 1893 (1975); [Sov. Phys. JETP 42, 961 (1976)].

[39] V. N. Băer, V. M. Katkov and V. M. Strakhovenko. "Electromagnetic processes at high energies in oriented single crystals.”, World Scientific, Singapore, (1998).

[40] W. Greiner and J. Reinhardt. "Quantum Electrodynamics.”, Springer, Berlin Heidelberg (2009).

[41] T. Heinzl, B. Leifeld, K. U. Amthor, H. Schwoerer, R. Sauerbrey and A. Wipf. Opt. Comm. 267, 318 (2006).

[42] Ben King, A. Di Piazza and C. H. Keitel. Nature 4, 92 (2010).

[43] S. Villalba-Chavez and A. E. Shabad in "QED with external field: Hamiltonian treatment for anisotropic medium formed by the Lorentznon-invariant vacuum"; arXiv:1206.4491 [hep-th].

[44] G. V. Dunne, in: M. Shifman, et al. (Eds.), From Fields to Strings: Circumnavigating Theoretical Physics, vol. 1 World Scientifics, Singapore, 2004, pp. 445-522; hep-th/0406216].

[45] I. S. Gradshteyn and I. M. Ryzhik. "Table of Integrals, Series and Products." Seventh Edition, Elsevier, San Diego, (2007).

[46] C. Schubert Nucl. Phys. B 585, 407 (2000); [hep-ph/0001288]. 